\title{
Association between Mental Disorders and Suicide
}

Bista $\mathbf{A B}^{1}$

${ }^{1} \mathrm{BPH}, \mathrm{CIST}$ College, Pokhara University

\section{Introduction}

A mental disorder or mental illness is a psychological aspect, which is mostly reflected in behavior, and is generally associated with distress or disability, and which is not considered part of normal development of a person's nature. Mental disorders are generally defined by a combination of how a person feels, acts, thinks or perceives. This may be associated with particular regions or functions of the brain or rest of the nervous system, often in a social context. (1) According to the World Health Organization (WHO), over a third of people in most countries report problems at some time in their life which meet criteria for diagnosis of one or more of the common types of mental disorder.

Although mental illness is a significant risk factor for suicide, the relationship between suicide and mental illness is complex. Mental illness alone does not cause suicidal behavior. People with mental illness may perceive the negative events of life and other difficult life circumstances in addition to the symptoms of their illness. Interaction of these all behavior finally leads to the suicidal behavior.

Suicide (Latinsuicidium, from sui caedere, "to kill oneself") is the act of intentionally causing one's own death. Suicide is often committed out of emotion, the cause of which can be attributed to a mental disorder such as depression, bipolar disorder, schizophrenia, autism spectrum disorders, alcoholism, or drug abuse.Over one million people die by suicide every year. The World Health Organization (WHO) estimates that it is the 13th leading cause of death worldwide It is a leading cause of death among teenagers and adults under 35. (2) The rate of suicide is far higher in men than in women, with males worldwide three to four times more likely to kill themselves than females. There are an estimated 10 to 20 million non-fatal attempted suicides every year worldwide.

\section{Risk factors}

A review found that $87 \%$ of the suicides where diagnosable with a mental disorder based on history from their friends and family following their death. and $27 \%$ of suicides in England between 2000 and 2010 had been in contact with mental health services in the year prior to their deaths. (3) There are a number of other factors correlated with suicide risk, including drug addiction, availability of means, family history of suicide, or previous head injury. Socio-economic factors such as unemployment, poverty, homelessness, and discrimination may trigger suicidal thoughts. (4) Poverty may not be a direct cause, but it can increase the risk of suicide, as impoverished individuals are a major risk group for depression. A history of childhood physical or sexual abuse is also a risk factor.

\section{Methods}

The literature review of previously researched journal and article by a well renowned person in related field and the associated organization is the sole effective for the preparation of the articles. Internet was used to retrieve the articles. For the purpose of reviewing articles, endnote software was used. After reviewing the corresponding article, hinari was used to retrieve the full version of articles. All together 10 full articles were downloaded. Google engine and Wikipedia was also a secondary component for the search of the articles. Some of the related books were also consulted. After the proper and thorough study of those related articles, this article was then finally prepared.

\section{Results}

Since there were not so many research made on relationship between suicide and mental disorder in Nepal, so the findings are mostly based on other countries. But the assumption is made that the research findings of other countries are also very much relevant with Nepal.

Now dealing with the research findings after the review of articles, certain mental disorders are often present at the time of suicide. It is estimated that from $87 \%$ to $98 \%$ of suicides are committed by people with some type of mental disorder. Broken down by type: mood disorders are present in $30 \%$, substance abuse in $18 \%$, schizophrenia in $14 \%$, and personality disorders in $13 \%$ of suicides. About $5 \%$ of people with schizophrenia die of suicide. Major depression and alcoholism are the specific disorders most strongly correlated with suicide risk. (5) Risk is greatest during the early stages of illness among people with mood disorders, such as major depression or bipolar disorder. One in five people with bipolar disorders complete suicide. Depression is among the most commonly diagnosed psychiatric disorders; increasingly diagnosed across various segments of the worldwide population. 17.6 million Americans are affected each year; approximately 1 in 6 people. Within the next twenty years, depression is expected to become the leading cause of disability in developed nations and the second leading cause of disability worldwide.A person's suicide risk increases again if the person has more than one mental illness. Studies in many countries have shown that medical students have higher levels of depression, anxiety and stress may be because of work load and the pressure during the education. 
So many medical students have found to be addicted in substance use and are more vulnerable group for suicide. (6) Not only among the medical students but also older group people have also major depressive disorders so the tendency however is not certain but is high among the elderly people. (7) $85 \%$ of the suicide was evaluated at Göteborg Institute of Forensic Medicine in a study and it was found that the sex and age distribution, proportion of certain suicides, and proportion of suicide cases that were found at postmortem analysis to have taken antidepressants were mostly elderly people.

\section{Discussion}

Suicide is the $13^{\text {th }}$ leading cause of death. Suicidal tendency, now a days is increasing rapidly and these all suicidal cases are associated to the emotional and mental state of any individual. Eventually mental disorders are also found to be the determinant factor of the suicide. The pain and distress associated to the mental disorders are so extreme that the people feel free to suicide so that they can get away of these all pain. Mental disorders like depression, schizophrenia, bipolar disorders and so on are associated to the suicide in high rate. Not only the mental disorders but also the substance uses, family history of suicide, hopelessness and sexual abuse is also related to the suicidal case. But among all these, $87 \%$ of the suicides where diagnosable with a mental disorder. Mental disorders are hard to diagnose at the initial phase because the symptoms initially are not so much strong so that they can be identified. But as it starts torturing the patient, the pain and distress is then high that person overwhelms the death than living a life.

\section{Conclusion}

After the proper literature review and all other findings, it can be concluded the suicide which is growing to be like a burden in health sector is highly associated with the mental disorders. The mental and psychological aspect is very much important determining factors of the suicide. Suicide is also associated with substance use, sexual assault, hopelessness. But among all those the suicide is highly correlated to the mental disorders which is found after the proper review of all those articles and books.

\section{Recommendation}

The situation which has been described above is not only the condition of other countries. Though the research to the related field in context of Nepal is few but the association cannot be neglected. The suicidal tendency is also increasing in Nepal. As public health student, I would like to recommend to all those concern authorities to take a serious and certain action and to get some statistics regarding the subject matter so that the possible cause of suicide can be identified and draw a proper policy guidelines. The mental hospitals should also be increased in number so that the diagnosis of mental illness can be made as soon as possible and the number of psychiatrist also be increased. The people should be made aware through different programs to visit psychiatrist at early phase if they notice any mental and emotional abnormalities so that the possible cure can be made very soon. Also the sexual assault and substance uses by the teenagers should need to be control. These are also other factors related to the suicide. Peer cooperation, family support and healthy natural environment needs to be develop to the patients of mental illness.

\section{References:}

1. The Fundamentals of Mental Health and Mental Illness." The United States Department of Health and Human Services. Mental Health: A Report of the Surgeon General.

2. O'Connor, R.S., Noel, Understanding suicidal behaviour, 29 Jan 2000

3. Arsenault-Lapierre G, K.C., Turecki G, "Psychiatric diagnoses in 3275 suicides: a meta-analysis". BMC Psychiatry, 2004

4. Wikipedia. 2012 [cited; Available from: www.wikipedia.com/ mental disorder.

5. Palmer BA, P.V., Bostwick JM, "The lifetime risk of suicide in schizophrenia: a reexamination". Arch. Gen. Psychiatry, March 2005

6. Zeynep Baykan, M.D., M.D. Melis Nac,ar, and P.D. Fevziye $\mathrm{C}$, etinkaya, Depression, Anxiety, and Stress Among LastYear Students at Erciyes University Medical School.

7. Preville, M., et al., Physical health and mental disorder in elderly suicide: a case-control study. Aging Ment Health, 2005. 9(6): p. 576-84. 